\title{
Quantum Interference in Jet Substructure from Spinning Gluons
}

\author{
Hao Chen $\oplus^{1, *}$ Ian Moult, ${ }^{2, \dagger}$ and Hua Xing Zhu $\odot^{1, \$}$ \\ ${ }^{1}$ Zhejiang Institute of Modern Physics, Department of Physics, Zhejiang University, Hangzhou 310027, China \\ ${ }^{2}$ SLAC National Accelerator Laboratory, Stanford University, California 94309, USA
}

(Received 13 November 2020; revised 11 February 2021; accepted 17 February 2021; published 17 March 2021)

\begin{abstract}
Collimated sprays of hadrons, called jets, are an emergent phenomenon of quantum chromodynamics (QCD) at collider experiments, whose detailed internal structure encodes valuable information about the interactions of high energy quarks and gluons and their confinement into color-neutral hadrons. The flow of energy within jets is characterized by correlation functions of energy flow operators, with the three-point correlator being the first correlator with nontrivial shape dependence, playing a special role in unraveling the dynamics of QCD. In this Letter, we initiate a study of the three-point energy correlator to all orders in the strong coupling constant, in the limit where two of the detectors are squeezed together. We show that, by rotating the two squeezed detectors with respect to the third by an angle $\phi, a \cos (2 \phi)$ dependence arising from the quantum interference between intermediate virtual gluons with $+/-$ helicity is imprinted on the detector. This can be regarded as a double slit experiment performed with jet substructure, and it provides a direct probe of the ultimately quantum nature of the substructure of jets and of transverse spin physics in QCD. To facilitate our all-orders analysis, we adopt the operator product expansion (OPE) for light-ray operators in conformal field theory and develop it in QCD. Our application of the light-ray OPE in real world QCD establishes it as a powerful theoretical tool with broad applications for the study of jet substructure.
\end{abstract}

DOI: 10.1103/PhysRevLett.126.112003

Introduction.-Jet substructure, which was originally developed to exploit the flow of energy within jets of particles at the Large Hadron Collider (LHC) to enhance new physics searches [1], has since emerged as a primary technique for studying QCD. By focusing on the internal structure of jets, one obtains a clean probe of the dynamics of QCD on the light cone, which is measured with exquisite angular precision by the LHC detectors. This has led to a wealth of theoretical and experimental progress with the goal of fully exploiting this remarkable dataset [2-4].

The flow of energy within jets is characterized by correlation functions $\left\langle\mathcal{E}\left(\hat{n}_{1}\right) \mathcal{E}\left(\hat{n}_{2}\right) \cdots \mathcal{E}\left(\hat{n}_{k}\right)\right\rangle$ of light-ray operators [5-12]

$$
\mathcal{E}(\hat{n})=\lim _{r \rightarrow \infty} \int_{0}^{\infty} d t r^{2} n^{i} T_{0 i}(t, r \hat{n}),
$$

in the limit where the $\hat{n}_{i}$ are nearly collinear, such that they lie within a single jet. Here $T_{\mu \nu}$ is the energy-momentum tensor, and $\hat{n}_{i}$ are unit vectors representing points (calorimeter cells) on the celestial sphere. Any observable measurable with detectors at infinity can be extracted from the complete set of such correlators $[5,6]$.

Published by the American Physical Society under the terms of the Creative Commons Attribution 4.0 International license. Further distribution of this work must maintain attribution to the author(s) and the published article's title, journal citation, and DOI. Funded by SCOAP ${ }^{3}$.
The simplest observable is the two-point correlator $[13,14]\left\langle\mathcal{E}\left(\hat{n}_{1}\right) \mathcal{E}\left(\hat{n}_{2}\right)\right\rangle$, which has no shape dependence. In the collinear limit, the two-point correlator exhibits a scaling behavior $[9,15-20]\left\langle\mathcal{E}\left(\hat{n}_{1}\right) \mathcal{E}\left(\hat{n}_{2}\right)\right\rangle \sim 1 / \theta_{12}^{2-2 \gamma\left(\alpha_{s}\right)}$. This scaling probes the value of the strong coupling constant $\alpha_{s}$ and has been computed to next-to-next-toleading logarithm in QCD [19].

Much more nontrivial information about the dynamics of QCD is encoded in higher-point correlation functions. In this Letter, we show that the squeezed limit of the threepoint correlator [21] $\left\langle\mathcal{E}\left(\hat{n}_{1}\right) \mathcal{E}\left(\hat{n}_{2}\right) \mathcal{E}\left(\hat{n}_{3}\right)\right\rangle$, where two detectors are brought together $\hat{n}_{2} \rightarrow \hat{n}_{3}$, is a direct probe of quantum interference between helicity $\lambda= \pm$ gluons in the jet, i.e., the transverse spin structure of $\mathrm{QCD}$, and that this is imprinted on the detector as a $\cos (2 \phi)$ interference pattern. While the study of transverse spin in QCD has a long history (see, e.g., [22-35]), the squeezed limit of the threepoint correlator provides a qualitatively new observable for studying transverse spin. Unlike standard probes of transverse spin that rely on nonperturbative fragmentation processes, energy correlators are infrared and collinear safe, and for the high energy jets at the LHC, exhibit a regime where the transverse spin structure can be computed in perturbative QCD. In the extreme collinear limit, they are sensitive to nonperturbative fragmentation, allowing for the study of the transition between perturbative and nonperturbative physics. Furthermore, they do not involve polarized beams or hadrons or reference directions and so are ideally suited for the LHC. 
The description of the complicated all-orders perturbative structure of multipoint correlators calls for the application of new theoretical tools. Much in analogy with how the local operator product expansion [36] $\mathcal{O}\left(x_{1}\right) \mathcal{O}\left(x_{2}\right)=\sum\left(x_{2}-x_{1}\right)^{\gamma_{i}} \mathcal{O}_{i}\left(x_{1}\right)$ describes the behavior of local operators as they are brought together, in the limit of interest in jet substructure where the light-ray operators $\mathcal{E}(\hat{n})$ become collinear, there exists a recently developed light-ray operator product expansion (OPE) $[9,12,17,37,38] \mathcal{E}\left(\hat{n}_{1}\right) \mathcal{E}\left(\hat{n}_{2}\right)=\theta^{\gamma_{i}} \sum \mathbb{O}_{i}\left(\hat{n}_{1}\right)$, where the $\mathbb{O}_{i}(\hat{n})$ are nonlocal light-ray operators [9,12,39-42]. However, the use of the light-ray OPE has so far been restricted to conformal field theories (CFTs) $[9,17,37,38]$.

In this Letter, we show that the light-ray OPE can be applied in QCD and provides powerful operator-based techniques for jet substructure. We show that the iterated OPE of $\mathcal{E}(\hat{n})$ operators closes at leading twist onto operators $\mathbb{O}_{i}^{[J]}(\hat{n})$ with arbitrary collinear spin $J$, but restricted transverse spin $j=0,2$, and we explicitly compute the $\mathcal{E}\left(\hat{n}_{1}\right) \mathcal{E}\left(\hat{n}_{2}\right)$ and $\mathbb{O}_{i}^{[J]}\left(\hat{n}_{1}\right) \mathcal{E}\left(\hat{n}_{2}\right)$ OPEs. The all-orders structure of spin interference effects in the threepoint correlator then arises naturally from the transverse spin structure of the light-ray OPE.

Interference in the squeezed limit.-The physics of the squeezed limit of the three-point correlator in a weakly coupled gauge theory can be described as a double slit experiment in spin space, see Fig. 1. The interference pattern in the usual double slit experiment is due to the interference in $\left|A_{L}(x)+A_{R}(x)\right|^{2}$, where $A_{L(R)}(x)$ is the amplitude for going through the left (right) slit from the light source to position $x$ on the detector. Similarly, in the squeezed limit of the three-point correlator, the interference terms in $\left|A_{+}(\phi)+A_{-}(\phi)\right|^{2}$ are the source of an interference pattern, where $A_{+(-)}$is the splitting amplitude with a nearly on-shell virtual gluon with positive (negative) helicity. Therefore, the slits in the standard double slit experiment are replaced by the intermediate $+/-$ helicity gluons, and varying the distance $x$ is replaced by varying the angle $\phi$ of the squeezed energy correlators. We emphasize that, while this effect arises from quantum interference, we have been unable to prove a Bell-type inequality using only energy measurements. It would be interesting to understand if Bell-type inequalities can be proven in the collider context, even in principle. Similar questions have also been considered in the context of inflationary measurements [43].

We parametrize the squeezed limit symmetrically, using $\left(\theta_{S}, \theta_{L}, \phi\right)$ as shown in Fig. 1, to eliminate linear power corrections in $\theta_{S} / \theta_{L}$. The squeezed limit is characterized by $\theta_{S} \ll \theta_{L}$, with $\phi$ arbitrary, and the expansion in this limit takes the form

$$
\frac{d^{3} \Sigma}{d \theta_{L}^{2} d \theta_{S}^{2} d \phi} \simeq \frac{1}{\pi}\left(\frac{\alpha_{s}}{4 \pi}\right)^{2} \frac{\mathrm{Sq}_{i}^{(0)}(\phi)}{\theta_{L}^{2} \theta_{S}^{2}}+\cdots,
$$

where the dots denote terms less singular in the squeezed limit. Expanding the full result for the three-point correlator in [21], we find for quark and gluon jets,

$$
\begin{aligned}
\mathrm{Sq}_{q}^{(0)}(\phi)= & C_{F} n_{f} T_{F}\left(\frac{39-20 \cos (2 \phi)}{225}\right) \\
& +C_{F} C_{A}\left(\frac{273+10 \cos (2 \phi)}{225}\right)+C_{F}^{2} \frac{16}{5} \\
= & 10.54+0.1156 n_{f} \\
& +\left(0.1778-0.0593 n_{f}\right) \cos (2 \phi), \\
\mathrm{Sq}_{g}^{(0)}(\phi)= & C_{A} n_{f} T_{F}\left(\frac{126-20 \cos (2 \phi)}{225}\right) \\
& +C_{A}^{2}\left(\frac{882+10 \cos (2 \phi)}{225}\right)+C_{F} n_{f} T_{F} \frac{3}{5} \\
= & \left(35.28+1.24 n_{f}\right)+\left(0.4-0.133 n_{f}\right) \cos (2 \phi) .
\end{aligned}
$$

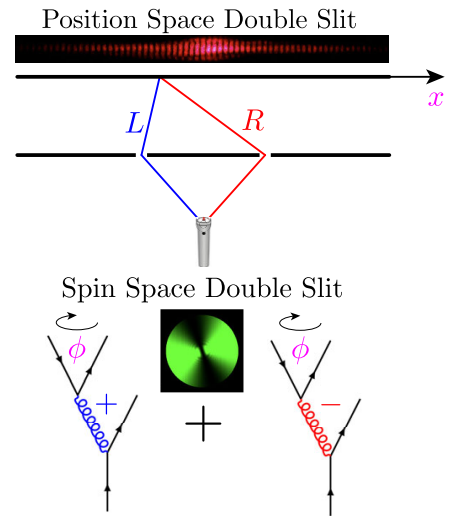

(a)

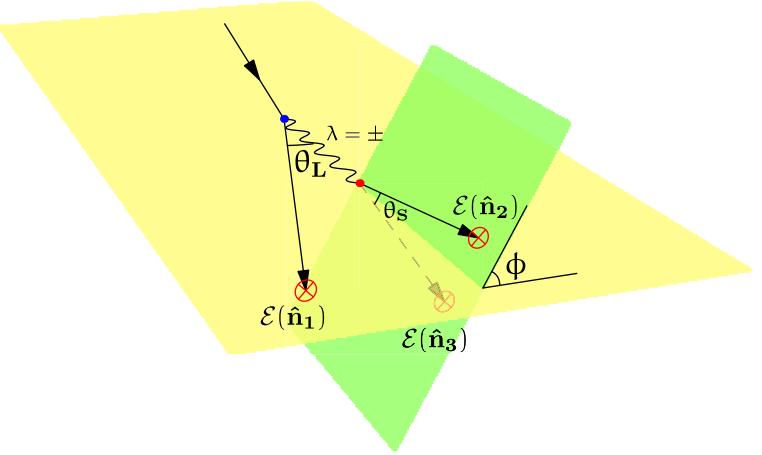

(b)

FIG. 1. (a) Double slit experiments in position and spin space and (b) physical implementation of the spin space double slit experiment using the squeezed limit $\theta_{S} \ll \theta_{L}$ of the three-point correlator. Quantum interference between gluon spin states $\lambda= \pm$ leads to a $\cos (2 \phi)$ pattern as the squeezed correlators are rotated. 
Here we see $\cos (2 \phi)$ interference terms at leading twist, which at this order are identical for quark and gluon jets, since they arise only from an intermediate gluon, and have opposite signs for $g \rightarrow q \bar{q}\left(n_{f} T_{F}\right.$ terms $)$ and $g \rightarrow g g$ $\left(C_{F} C_{A}\right.$ and $C_{A}^{2}$ terms). Positivity of the cross section guarantees that the $\cos (2 \phi)$ terms are smaller than the constant terms, analogous to the conformal collider bounds [9]. Because of the singular structure of the squeezed limit, the all-orders resummation of these spin interference effects is required to describe the three-point correlator, as well as for limits of higher-point correlators.

Despite their importance for observables relevant to jet substructure, spin interference effects are not included in the standard parton shower simulations used to this point by experiments at the LHC, which, as illustrated through detailed studies in [44], can lead to large errors for multiemission jet substructure observables. Recently, significant progress was made with the implementation of algorithms for including spin interference effects in parton showers [45-50] in a new version of Herwig [50,51]. The goal of this Letter will be to understand the all-orders structure of these spin interference effects analytically and show how they emerge in a simple manner from the transverse spin structure of the lightray OPE.

The light-ray OPE in $Q C D$.-To our knowledge, the analytic resummation of spin interference effects in the collinear limit has not been achieved using standard perturbative QCD techniques. This is in contrast to the more well-studied Sudakov (or back-to-back) region (see, e.g., $[52,53])$. To achieve this resummation, we will apply the light-ray OPE formalism $[9,12,17,37,38]$. In addition to this particular phenomenological application, our calculation provides a highly nontrivial application of the light-ray OPE in a nonconformal field theory and illustrates its potential for jet substructure at the LHC.

The OPE of light-ray operators was originally presented [9] in the context of gauge theories using the string operators of [39]. It has recently been developed into a rigorous OPE in CFTs $[12,17,37,38]$. Although the loss of symmetries relaxes the rigid structure of the OPE in a CFT, the intuition for its existence remains. Furthermore, it is well known that QCD often inherits structure from the conformal limit [42].

For multiple light-ray operators with small transverse separations, one expects an OPE onto a sum of light-ray operators each formed from potentially multiple operators smeared on the light cone, as shown in the Penrose diagram in Fig. 2. Characterizing the operators involved by their twist, collinear spin $J$ and transverse spin $j$, in a weakly coupled gauge theory, such as QCD in the perturbative regime, we have the simplification that the leading twist contributions involve two fields and therefore can only have transverse spin 0 or 2 . This greatly simplifies the OPE,

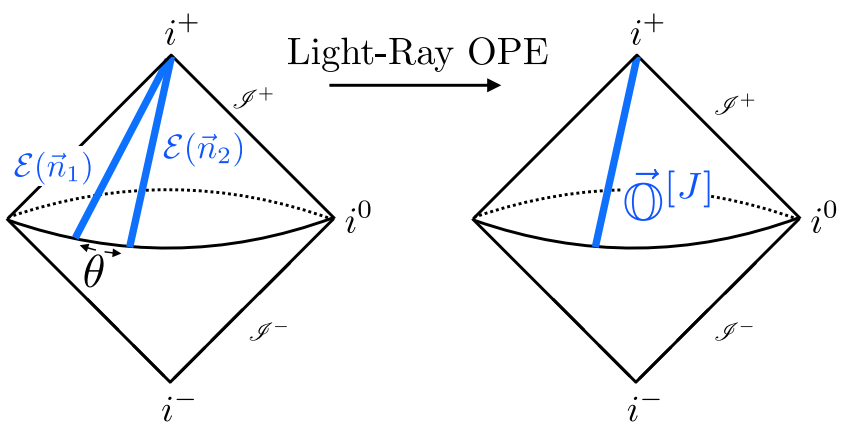

FIG. 2. At leading twist, the light-ray OPE in QCD for $\mathcal{E}$ operators leads to the light-ray operators $\overrightarrow{\mathbb{O}}^{[J]}$ characterized by a spin $J$ and transverse spin $j=0,2$.

allowing it to be iterated to any number of light-ray operators. The structure beyond leading twist is known for the $\mathcal{E}\left(\hat{n}_{1}\right) \mathcal{E}\left(\hat{n}_{2}\right)$ OPE in CFTs [38].

The leading twist operators in QCD are (see, e.g., [54])

$$
\begin{aligned}
\mathcal{O}_{q}^{[J]} & =\frac{1}{2^{J}} \bar{\psi} \gamma^{+}\left(i D^{+}\right)^{J-1} \psi, \\
\mathcal{O}_{g}^{[J]} & =-\frac{1}{2^{J}} F_{a}^{\mu+}\left(i D^{+}\right)^{J-2} F_{a}^{\mu+}, \\
\mathcal{O}_{\tilde{g}, \lambda}^{[J]} & =-\frac{1}{2^{J}} F_{a}^{\mu+}\left(i D^{+}\right)^{J-2} F_{a}^{\nu+} \epsilon_{\lambda, \mu} \epsilon_{\lambda, \nu},
\end{aligned}
$$

where the plus component of a vector $A^{\mu}$ is $\bar{n} \cdot A$, and $\bar{n}=(1,-\hat{n})$. We have projected the transverse spin-2 operators onto helicities $\lambda= \pm$ using polarization vectors $\epsilon^{\mu}$. These transverse spin-2 operators are purely gluonic and will reproduce the interference terms in Eq. (3). Smearing on the light cone (the light transform [12]) gives rise to light-ray operators, which we write as a vector

$$
\overrightarrow{\mathbb{O}}^{[J]}=\left(\mathbb{O}_{q}^{[J]}, \mathbb{O}_{g}^{[J]}, \mathbb{O}_{\tilde{g},+}^{[J]}, \mathbb{O}_{\tilde{g},-}^{[J]}\right)^{T}=\lim _{r \rightarrow \infty} r^{2} \int_{0}^{\infty} d t \overrightarrow{\mathcal{O}}^{[J]}(t, r \vec{n}) .
$$

To derive the leading twist $\mathcal{E}\left(\hat{n}_{1}\right) \mathcal{E}\left(\hat{n}_{2}\right)$ OPE in the leading logarithmic approximation, we perform an explicit matching calculation using Wightman functions. Since the OPE is state independent, we compute the OPE coefficients by performing a matching calculation using states produced by gauge invariant collinear quark or gluon fields. At lowest order in perturbation theory, the diagrams entering the matching calculation describe the splitting of the quark or gluon field into the two energy detectors. We find

$$
\begin{aligned}
\mathcal{E}\left(\hat{n}_{1}\right) \mathcal{E}\left(\hat{n}_{2}\right)= & -\frac{1}{2 \pi} \frac{2}{\theta^{2}} \overrightarrow{\mathcal{J}}\left[\hat{C}_{\phi}(2)-\hat{C}_{\phi}(3)\right] \overrightarrow{\mathbb{O}}^{[3]}\left(\hat{n}_{1}\right) \\
& + \text { higher twist, }
\end{aligned}
$$

where $\theta$ is the angle between the OPE pair, and $\overrightarrow{\mathcal{J}}=$ $(1,1,0,0)$ is a projector onto unpolarized quark and gluon twist operators, and the matrix of OPE coefficients is 


$$
\hat{C}_{\phi}(J)=\left(\begin{array}{cc}
\gamma_{q q}(J) & 2 n_{f} \gamma_{q g}(J) \\
\gamma_{g q}(J) & \gamma_{g g}(J) \\
\gamma_{\tilde{g} q}(J) e^{2 i \phi} & \gamma_{\tilde{g} g}(J) e^{2 i \phi} \\
\gamma_{\tilde{g} q}(J) e^{-2 i \phi} & \gamma_{\tilde{g} g}(J) e^{-2 i \phi}
\end{array}\right.
$$

The quantum number $J=3$ appearing in Eq. (5) arises from the gauge theory perspective due to the fact that the two detectors lead to an $E^{2}$ weighting of the splitting function, corresponding to its third moment [19], while from a CFT perspective, it is fixed by the quantum numbers of the light-ray operators [9].

While the general structure of this OPE was anticipated in $[9,38]$, the explicit form of the OPE in QCD is a new result. An interesting feature of Eq. (6) is that the OPE coefficients of light-ray operators are themselves anomalous dimensions. Indeed, $\gamma_{i j}(J), i, j=q, g$ are the standard twist-2 spin- $J$ anomalous dimensions, related to the unpolarized splitting functions, while $\gamma_{i, \tilde{g}}(J), i=q, g$ are moments of the spin correlated splitting functions of [55]. This builds a direct bridge between the language of splitting functions in perturbative gauge theories and the light-ray OPE, which deserves further exploration. On the other hand, $\gamma_{\tilde{g}, i}(J)$ have, to our knowledge, not appeared previously in the QCD literature. They do not appear in the $\mathcal{E}\left(\hat{n}_{1}\right) \mathcal{E}\left(\hat{n}_{2}\right)$ OPE due to the projector $\overrightarrow{\mathcal{J}}$, but will appear in the more general $\mathbb{O}^{[J]}\left(\hat{n}_{1}\right) \mathcal{E}\left(\hat{n}_{2}\right)$ OPE considered below. Denoting the perturbative expansion as $\gamma_{i j}=\left[\alpha_{s} /(4 \pi)\right] \gamma_{i j}^{(0)}+\mathcal{O}\left(\alpha_{s}^{2}\right)$, we have

$\gamma_{\tilde{g} \tilde{g}}^{(0)}(J)=4 C_{A}\left[\psi^{(0)}(J+1)+\gamma_{E}\right]-\beta_{0}$,

$\gamma_{\tilde{g} q}^{(0)}(J)=C_{F} \frac{2}{(J-1) J}, \quad \gamma_{\tilde{g} g}^{(0)}(J)=C_{A} \frac{2}{(J-1) J}$,

$\gamma_{q \tilde{g}}^{(0)}(J)=-T_{F} \frac{8}{(J+1)(J+2)}, \quad \gamma_{\tilde{g} \tilde{g}, \pm}^{(0)}(J)=0$,

$\gamma_{g \tilde{g}}^{(0)}(J)=C_{A}\left(\frac{8}{(J+1)(J+2)}+3\right)-\beta_{0}$,

where $\psi^{(0)}$ is the digamma function, and $\beta_{0}=11 C_{A} / 3-$ $4 T_{F} n_{f} / 3$ is the first-order QCD $\beta$ function. The other anomalous dimensions are standard (see, e.g., [20]).

Plugging the explicit values of the OPE coefficients in Eq. (6) for $J=3$ into the $\mathcal{E}\left(\hat{n}_{1}\right) \mathcal{E}\left(\hat{n}_{2}\right)$ OPE of Eq. (5) reproduces the result for the two-point correlator in QCD for both quark and gluon sources [19], providing a nontrivial check of Eq. (5).

In a CFT, the collinear spin $J=3$ appearing in the $\mathcal{E}\left(\hat{n}_{1}\right) \mathcal{E}\left(\hat{n}_{2}\right)$ OPE is fixed by symmetry $[9,17]$. From the structure of higher logarithmic terms in [19], we expect that in QCD light-ray operators with $J=3+\mathcal{O}\left(\alpha_{s}\right)$ appear at

$$
\left.\begin{array}{cc}
2 n_{f} \gamma_{q \tilde{g}}(J) e^{-2 i \phi} / 2 & 2 n_{f} \gamma_{q \tilde{g}}(J) e^{2 i \phi} / 2 \\
\gamma_{g \tilde{g}}(J) e^{-2 i \phi} / 2 & \gamma_{g \tilde{g}}(J) e^{2 i \phi} / 2 \\
\gamma_{\tilde{g} \tilde{g}}(J) & \gamma_{\tilde{g} \tilde{g}, \pm}(J) e^{4 i \phi} \\
\gamma_{\tilde{g} \tilde{g}, \pm}(J) e^{-4 i \phi} & \gamma_{\tilde{g} \tilde{g}}(J)
\end{array}\right) .
$$

higher orders due to the breaking of conformal symmetry and the nontrivial manifestation of Basso-Korchemsky reciprocity [56,57] in the energy-energy correlator [19].

Squeezed limits from light-ray OPEs.-To compute the squeezed limit of the three-point correlator $\left\langle\mathcal{E}\left(\hat{n}_{1}\right) \mathcal{E}\left(\hat{n}_{2}\right)\right.$ $\left.\mathcal{E}\left(\hat{n}_{3}\right)\right\rangle$, we perform the successive OPE $\mathcal{E}\left(\hat{n}_{2}\right) \mathcal{E}\left(\hat{n}_{3}\right) \rightarrow$ $\sum \mathbb{O}^{[3]}\left(\hat{n}_{2}\right)$ followed by $\mathcal{E}\left(\hat{n}_{1}\right) \overrightarrow{\mathbb{O}}^{[J]}\left(\hat{n}_{2}\right) \rightarrow \overrightarrow{\mathbb{O}}^{[J+1]}\left(\hat{n}_{1}\right)$. The second OPE is computed analogously to the $\mathcal{E}\left(\hat{n}_{1}\right) \mathcal{E}\left(\hat{n}_{2}\right)$ OPE discussed above. Because the maximal transverse spin at leading twist in QCD is two, we find that the iterated OPE of light-ray operators closes onto the operators $\overrightarrow{\mathbb{O}}^{[J]}\left(\hat{n}_{1}\right)$. To be able to apply our results to arbitrary iterated squeezed limits, we compute the OPE coefficients in the $\mathcal{E}\left(\hat{n}_{1}\right) \overrightarrow{\mathbb{O}}^{[J]}\left(\hat{n}_{2}\right)$ OPE as analytic functions of the collinear spin $J$.

The $\overrightarrow{\mathbb{O}}^{[J]}\left(\hat{n}_{1}\right) \mathcal{E}\left(\hat{n}_{2}\right)$ OPE is

$$
\begin{aligned}
\overrightarrow{\mathbb{O}}^{[J]}\left(\hat{n}_{1}\right) \mathcal{E}\left(\hat{n}_{2}\right)= & -\frac{1}{2 \pi} \frac{2}{\theta^{2}}\left[\hat{C}_{\phi}(J)-\hat{C}_{\phi}(J+1)\right] \overrightarrow{\mathbb{O}}^{[J+1]}\left(\hat{n}_{1}\right) \\
& + \text { higher twist. }
\end{aligned}
$$

Unlike the $\mathcal{E}\left(\hat{n}_{1}\right) \mathcal{E}\left(\hat{n}_{2}\right)$ OPE, this involves the full matrix of OPE coefficients, including the entries $\gamma_{\tilde{g}, i}(J)$.

In the squeezed limit, the hierarchy between the splitting angles $\theta_{S} \ll \theta_{L}$ gives rise to large logarithmic corrections in OPE coefficients that must be resummed to all orders to obtain a physical result. This resummation is performed by solving the renormalization group equations for the lightray operators

$$
\frac{d}{d \ln \mu^{2}} \overrightarrow{\mathbb{O}}^{[J]}=-\hat{\gamma}(J) \cdot \overrightarrow{\mathbb{O}}^{[J]},
$$

where

$$
\hat{\gamma}(J)=\left(\begin{array}{ccc}
\gamma_{q q}(J) & 2 n_{f} \gamma_{q g}(J) & 0 \\
\gamma_{g q}(J) & \gamma_{g g}(J) & 0 \\
0 & 0 & \gamma_{\tilde{g} \tilde{g}}(J) \mathbf{1}
\end{array}\right),
$$

where $\mathbf{1}$ is a $2 \times 2$ identity matrix. Combining Eqs. (5), (8), and (9), we derive a resummed result describing the leading twist singular behavior in the squeezed limit of the threepoint correlator at leading logarithmic order 
$\mathcal{E}\left(\hat{n}_{1}\right) \mathcal{E}\left(\hat{n}_{2}\right) \mathcal{E}\left(\hat{n}_{3}\right)=\frac{1}{(2 \pi)^{2}} \frac{2}{\theta_{S}^{2}} \frac{2}{\theta_{L}^{2}} \overrightarrow{\mathcal{J}}\left[\hat{C}_{\phi_{S}}(2)-\hat{C}_{\phi_{S}}(3)\right]\left[\frac{\alpha_{s}\left(\theta_{L} Q\right)}{\alpha_{s}\left(\theta_{S} Q\right)}\right]^{\frac{\gamma^{(0)(3)}}{\beta_{0}}}\left[\hat{C}_{\phi_{L}}(3)-\hat{C}_{\phi_{L}}(4)\right]\left[\frac{\alpha_{s}(Q)}{\alpha_{s}\left(\theta_{L} Q\right)}\right]^{\frac{\gamma^{(0)(4)}}{\beta_{0}}} \overrightarrow{\mathbb{O}}^{[4]}\left(\hat{n}_{1}\right)+\cdots$

where the azimuthal angle $\phi$ in Eq. (2) is identified as $\phi_{S}-\phi_{L}$, and the overall rotation of the jet can be integrated out since we consider unpolarized sources. The dots denote higher twist and subleading logarithmic contributions. Plugging in the explicit values for the anomalous dimensions, Eq. (7), and expanding to leading order in $\alpha_{s}$, we find that Eq. (11) exactly reproduces the fixed-order results in Eq. (3), providing a highly nontrivial test of our OPE formulas.

We can also consider the squeezed limit of the threepoint correlator in $\mathcal{N}=4$ supersymmetric Yang-Mills (SYM) theory [21]. Here, the evolution matrix in Eq. (10), reduces to a scalar evolution with a universal anomalous dimension [58], and $\gamma_{\tilde{g} \tilde{g}}=\gamma_{g g}[9,59]$, so Eq. (11) agrees with the prediction for the scaling of the transverse spin-0 and spin-2 contributions from [9]. However, we find that the leading twist spin correlations vanish after summing over the multiplet, since $\sum\left[C_{\phi}(3)\right]_{i, \tilde{g}}=0, i=\phi, q, g$. This agrees with the perturbative prediction of [21]. This is one manifestation of the "classicality" of $\mathcal{N}=4$ SYM.

Numerical results at the LHC.-Using Eq. (11), we make numerical predictions for unpolarized quark and gluon jets at the LHC. In Figs. 3(a) and 3(b), we show the squeezed limit of the three-point correlator, weighted by

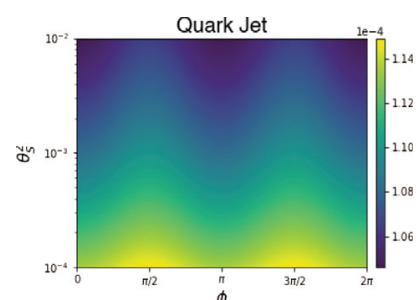

(a)

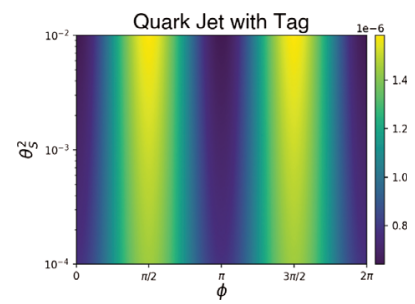

(c)

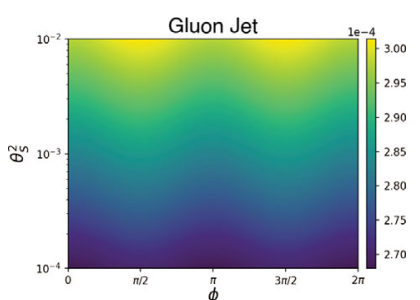

(b)

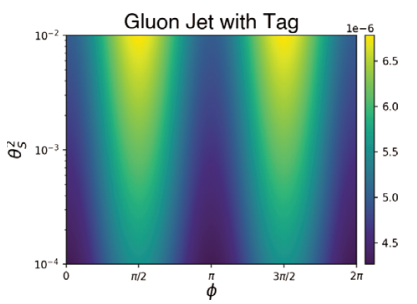

(d)
FIG. 3. The squeezed limit of the three-point correlator for (a) quark and (b) gluon jets at the LHC. Interference effects are seen as the $\cos (2 \phi)$ ripple modulated by resummation in $\theta_{S}$. (c), (d) We assume an idealized $b$ tagger, as explained in the text, to enhance the effect.
$\theta_{L}^{2} \theta_{S}^{2}$, as a function of $\left(\phi, \theta_{S}\right)$ for fixed $\theta_{L}$. Here we have used $Q=1 \mathrm{TeV}, \theta_{L}^{2}=0.1$, and $\alpha_{s}(1 \mathrm{TeV})=0.087$, as well as the one-loop $\beta$ function. The ripples in the distribution are clearly visible and illustrate the direct imprint of quantum interference effects in the detector. They are modulated by the resummation in $\theta_{S}$, which has a qualitatively different structure for quark and gluon jets, as discussed in [19].

For the case of QCD with $n_{f}=5$ light flavors, the interference effects are at the few percent level due to a cancellation of $g \rightarrow q \bar{q}$ and $g \rightarrow g g$ splittings. However, we believe that if measured using tracks, they are on the boundary of what can be achieved (see, e.g., [60]). Furthermore, there are a number of ways to enhance the interference signals, including using charge information to identify the $g \rightarrow q q$ splitting or $b$ tagging to perform the measurement. In Figs. 3(c) and 3(d), we show predictions using an idealized $b$ tagging on the squeezed pair, which isolates the $g \rightarrow b b$ contribution and enhances the modulation to an $\mathcal{O}(1)$ effect. We leave a detailed phenomenological study of the optimal strategy to future work.

Conclusions. - In this Letter, we have studied the threepoint energy correlator $\left\langle\mathcal{E}\left(\hat{n}_{1}\right) \mathcal{E}\left(\hat{n}_{2}\right) \mathcal{E}\left(\hat{n}_{3}\right)\right\rangle$ at the LHC. Our study is novel both phenomenologically, where we have proposed to use squeezed limits of energy correlators to probe quantum interference and transverse spin effects in jet substructure, as well as theoretically, where we have developed the light-ray OPE in QCD, and showed that it provides a transparent way of understanding the resummation of spin interference effects.

Our results for the $\mathcal{E}\left(\hat{n}_{1}\right) \mathcal{E}\left(\hat{n}_{2}\right)$ and $\mathcal{E}\left(\hat{n}_{1}\right) \mathbb{O}^{[J]}\left(\hat{n}_{2}\right)$ OPEs in QCD allow the description of iterated squeezed limits of $n$-point correlators and opens the door for these observables to be used as precision probes of QCD at the LHC. The measurement of these multipoint event correlators in the deep nonperturbative regime is also fascinating as they are sensitive to polarization effects in the nonperturbative fragmentation process.

There are numerous avenues for further theoretical development of the light-ray OPE in QCD, including understanding the appearance of light-ray operators with noninteger spin at higher logarithmic orders, the structure of the $1 \rightarrow 3$ light-ray OPE, and the inclusion of electromagnetically charged correlators [61]. Furthermore, many techniques developed to exploit symmetries in the CFT setting, such as the use of conformal blocks to resum higher twist corrections $[17,38]$ or the use of differential operators to relate contributions with different quantum numbers [62], can be applied in the QCD context to significantly 
extend standard splitting-function-based approaches. We therefore believe that the application of the light-ray OPE to problems of phenomenological interest at the LHC can lead to fruitful collaboration between the CFT and jet substructure communities, leading to significant advances in our understanding of QCD.

We thank Ming-xing Luo, Tong-Zhi Yang, and XiaoYuan Zhang for helpful discussions about energy correlators in perturbation theory, David Simmons-Duffin, Petr Kravchuk, and Cyuan-Han Chang for helpful discussions about energy correlators and the light-ray OPE, Jesse Thaler and Patrick Komiske for helpful discussions about aspects of energy correlators at the LHC, and Duff Neill for helpful discussions about the nuclear physics literature. We thank Alexander Karlberg for helpful comments on the Letter. H. C. and H.X.Z. are supported by National Natural Science Foundation of China under Contract No. 11975200. I. M. is supported by the Office of High Energy Physics of the U.S. DOE under Award No. DEAC02-76SF00515. H. X. Z. acknowledges the support of the Qiu Shi Science and Technologies Foundation.

*chenhao201224@zju.edu.cn

†imoult@slac.stanford.edu

zhuhx@zju.edu.cn

[1] J. M. Butterworth, A. R. Davison, M. Rubin, and G. P. Salam, Phys. Rev. Lett. 100, 242001 (2008).

[2] A. J. Larkoski, I. Moult, and B. Nachman, Phys. Rep. 841, 1 (2020).

[3] R. Kogler, B. Nachman, A. Schmidt, L. Asquith, M. Campanelli, C. Delitzsch, P. Harris, A. Hinzmann, D. Kar, C. McLean J. Pilot, Y. Takahashi, N. Tran, C. Vernieri, and M. Vos, Rev. Mod. Phys. 91, 045003 (2019).

[4] S. Marzani, G. Soyez, and M. Spannowsky, Lect. Notes Phys. 958, (2019).

[5] N. A. Sveshnikov and F. V. Tkachov, Phys. Lett. B 382, 403 (1996).

[6] F. V. Tkachov, Int. J. Mod. Phys. A 12, 5411 (1997).

[7] G. P. Korchemsky and G. F. Sterman, Nucl. Phys. B555, 335 (1999).

[8] C. W. Bauer, S. P. Fleming, C. Lee, and G. F. Sterman, Phys. Rev. D 78, 034027 (2008).

[9] D. M. Hofman and J. Maldacena, J. High Energy Phys. 05 (2008) 012.

[10] A. V. Belitsky, S. Hohenegger, G. P. Korchemsky, E. Sokatchev, and A. Zhiboedov, Nucl. Phys. B884, 305 (2014).

[11] A. V. Belitsky, S. Hohenegger, G. P. Korchemsky, E. Sokatchev, and A. Zhiboedov, Nucl. Phys. B884, 206 (2014).

[12] P. Kravchuk and D. Simmons-Duffin, J. High Energy Phys. 11 (2018) 102.

[13] C. L. Basham, L. S. Brown, S. D. Ellis, and S. T. Love, Phys. Rev. Lett. 41, 1585 (1978).

[14] C. L. Basham, L. S. Brown, S. D. Ellis, and S. T. Love, Phys. Rev. D 19, 2018 (1979).
[15] K. Konishi, A. Ukawa, and G. Veneziano, Nucl. Phys. B157, 45 (1979).

[16] D. G. Richards, W. J. Stirling, and S. D. Ellis, Phys. Lett. 119B, 193 (1982).

[17] M. Kologlu, P. Kravchuk, D. Simmons-Duffin, and A. Zhiboedov, J. High Energy Phys. 01 (2021) 128.

[18] G. P. Korchemsky, J. High Energy Phys. 01 (2020) 008.

[19] L. J. Dixon, I. Moult, and H. X. Zhu, Phys. Rev. D 100, 014009 (2019).

[20] H. Chen, I. Moult, X. Y. Zhang, and H. X. Zhu, Phys. Rev. D 102, 054012 (2020).

[21] H. Chen, M. X. Luo, I. Moult, T. Z. Yang, X. Zhang, and H. X. Zhu, J. High Energy Phys. 08 (2020) 028.

[22] A. V. Efremov and O. V. Teryaev, Sov. J. Nucl. Phys. 36, 140 (1982).

[23] A. V. Efremov and O. V. Teryaev, Phys. Lett. 150B, 383 (1985).

[24] J. w. Qiu and G. F. Sterman, Phys. Rev. Lett. 67, 2264 (1991).

[25] J. C. Collins, Nucl. Phys. B396, 161 (1993).

[26] J. C. Collins, S. F. Heppelmann, and G. A. Ladinsky, Nucl. Phys. B420, 565 (1994).

[27] J. C. Collins, Phys. Lett. B 536, 43 (2002).

[28] A. Airapetian et al. (HERMES Collaboration), Phys. Rev. Lett. 94, 012002 (2005).

[29] D. Boer, R. Jakob, and P. J. Mulders, Nucl. Phys. B504, 345 (1997).

[30] J. Qiu and G. Sterman, Phys. Rev. D 59, 014004 (1998).

[31] R. Seidl et al. (Belle Collaboration), Phys. Rev. D 78, 032011 (2008); 86, 039905(E) (2012).

[32] V. Barone, F. Bradamante, and A. Martin, Prog. Part. Nucl. Phys. 65, 267 (2010).

[33] J. Collins, Cambridge Monogr. Part. Phys., Nucl. Phys., Cosmol. 32, 1 (2011).

[34] Z. B. Kang, A. Prokudin, P. Sun, and F. Yuan, Phys. Rev. D 93, 014009 (2016).

[35] Z. B. Kang, K. Lee, and F. Zhao, Phys. Lett. B 809, 135756 (2020).

[36] K. G. Wilson, Phys. Rev. 179, 1499 (1969).

[37] M. Kologlu, P. Kravchuk, D. Simmons-Duffin, and A. Zhiboedov, J. High Energy Phys. 11 (2020) 096.

[38] C. H. Chang, M. Kologlu, P. Kravchuk, D. SimmonsDuffin, and A. Zhiboedov, arXiv:2010.04726.

[39] I. I. Balitsky and V. M. Braun, Nucl. Phys. B311, 541 (1989).

[40] I. I. Balitsky and V. M. Braun, Phys. Lett. B 222, 123 (1989).

[41] I. I. Balitsky and V. M. Braun, Nucl. Phys. B361, 93 (1991).

[42] V. M. Braun, G. P. Korchemsky, and D. Müller, Prog. Part. Nucl. Phys. 51, 311 (2003).

[43] J. Maldacena, Fortschr. Phys. 64, 10 (2016).

[44] M. Dasgupta, F. A. Dreyer, K. Hamilton, P. F. Monni, G. P. Salam, and G. Soyez, Phys. Rev. Lett. 125, 052002 (2020).

[45] J. C. Collins, Nucl. Phys. B304, 794 (1988).

[46] I. G. Knowles, Comput. Phys. Commun. 58, 271 (1990).

[47] I. G. Knowles, Nucl. Phys. B310, 571 (1988).

[48] I. G. Knowles, Nucl. Phys. B304, 767 (1988).

[49] P. Richardson, J. High Energy Phys. 11 (2001) 029.

[50] P. Richardson and S. Webster, Eur. Phys. J. C 80, 83 (2020).

[51] J. Bellm, G. Bewick, S. Ferrario Ravasio, S. Gieseke, D. Grellscheid, P. Kirchgaeßer, M. R. Masouminia, G. Nail, A. Papaefstathiou, S. Platzer et al., Eur. Phys. J. C 80, 452 (2020). 
[52] Y. T. Chien, R. Rahn, S. Schrijnder van Velzen, D. Y. Shao, W. J. Waalewijn, and B. Wu, Phys. Lett. B 815, 136124 (2021).

[53] Y. Hatta, B. W. Xiao, F. Yuan, and J. Zhou, arXiv:2010.10774.

[54] N. H. Christ, B. Hasslacher, and A. H. Mueller, Phys. Rev. D 6, 3543 (1972).

[55] D. de Florian and M. Grazzini, Phys. Rev. Lett. 85, 4678 (2000).

[56] B. Basso and G. P. Korchemsky, Nucl. Phys. B775, 1 (2007).

[57] H. Chen, T.Z. Yang, H.X. Zhu, and Y.J. Zhu, arXiv:2006.10534.
[58] A. V. Kotikov, L. N. Lipatov, A. I. Onishchenko, and V. N. Velizhanin, Phys. Lett. B 595, 521 (2004); 632, 754(E) (2006).

[59] N. Beisert, Nucl. Phys. B676, 3 (2004).

[60] G. Aad et al. (ATLAS Collaboration), Phys. Rev. D 101, 052007 (2020).

[61] D. Chicherin, J. M. Henn, E. Sokatchev, and K. Yan, J. High Energy Phys. 02 (2021) 053.

[62] D. Karateev, P. Kravchuk, and D. Simmons-Duffin, J. High Energy Phys. 02 (2018) 081. 